\title{
Polymers Interfacing with Biology
}

\author{
Frederik Wurm and Harm-Anton Klok*
}

\begin{abstract}
The development of various controlled radical polymerization techniques as well as site- and residue specific strategies to modify peptides/proteins with synthetic polymers have made polymer chemistry a powerful tool to address materials problems at the biology interface. This article will present recent examples for bioactive surface modification and polymer therapeutics; it will highlight the use of controlled radical polymerization techniques and bioconjugation strategies to develop surface coatings for regenerative medicine and diagnostics, respectively, polymer-based nanomedicines.
\end{abstract}

Keywords: Biopolymers · PEGylation · Polymer brush · Polymer therapeutics · Surface modification

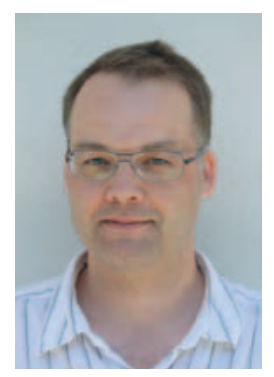

\section{Introduction}

Controlling the interactions between synthetic materials and biology is a crucial issue for the development of new materials for therapeutics, regenerative medicine or diagnostic purposes. Examples include the specific modification of peptide/protein drugs with synthetic polymers to allow targeted delivery and prolong plasma half-lives, the modification of implantable devices with bioactive coatings that avoid a foreign body response and high capacity protein or DNA binding surface coatings for biodiagnostics. These challenges call for synthetic strategies that allow precise control over polymer molecular weight, molecular weight distribution, functionality and architecture as well as for methods that enable site and/or residue specific conjugation of synthetic polymers to peptides, proteins or other biomolecules. Among various other advances, in particular the development of various controlled/'living' radical polymerization techniques as well

${ }^{\star}$ Correspondence: Prof. Dr. H.-A. Klok

Ecole Polytechnique Fédérale de Lausanne (EPFL) Institut des Matériaux and Institut des Sciences et Ingénierie Chimiques

Laboratoire des Polymères, Bâtiment MXD, Station 12, $\mathrm{CH}-1015$ Lausanne

Tel.: +41216934866

Fax: +41216935650

E-mail: harm-anton.klok@epfl.ch as site- and residue specific strategies to modify peptides/proteins with synthetic polymers have made polymer chemistry a powerful tool to address materials problems at the biology interface. Using examples from the authors' own research activities, this article seeks to illustrate some of these advances and successively will highlight the use of controlled radical polymerization techniques and bioconjugation strategies to develop surface coatings for regenerative medicine and diagnostics, respectively, polymer-based nanomedicines.

\section{Regenerative Medicine and Diagnostics}

Successful use of implantable medical devices or scaffolds to allow in vivo tissue regeneration requires control over cell-surface interactions. One strategy to achieve this involves modifying the surface of the substrate of interest with a nonbiofouling polymer coating, i.e. a coating that is non-adhesive to proteins and cells, but which can be functionalized with specific biochemical cues to interact with specific cells. Non-biofouling coatings are typically composed of a thin layer of a hydrophilic polymer that can be prepared via various strategies. Surface-initiated controlled radical polymerization techniques are particularly attractive as they allow access to thin surface-grafted polymer layers, so-called polymer brushes, with relatively accurate control over thickness, composition and architecture. ${ }^{[1]}$ To illustrate this approach, Fig. 1 outlines the synthesis of RGD-functionalized poly(2hydroxyethyl methacrylate) (PHEMA) or poly(polyethylene glycol methacrylate) (PPEGMA) brushes that were used as substrates for the integrin specific adhesion of human umbilical vascular endothelial cells (HUVECs). PHEMA and PPEGMA brushes with thicknesses between 20 and $150 \mathrm{~nm}$ were synthesized via surface-ini- tiated atom transfer radical polymerization (SI-ATRP), activated by reaction with $p$ nitrophenyl chloroformate and finally postmodified with the RGD-peptide (Fig. 1). [2] The peptide modification was monitored via FTIR-spectroscopy (appearance of the amide bands) and X-ray photoelectron spectroscopy (appearance of nitrogen signals). A peptide surface concentration between 0.5 and $12 \mathrm{pmol} / \mathrm{cm}^{2}$ was estimated by the use of a pyrene-labeled peptide and analysis via UV-vis spectroscopy. When incubated with HUVECs, all peptidemodified brushes showed enhanced cell adhesion and proliferation above a threshold RGD-surface concentration of 1-5.3 $\mathrm{pmol} / \mathrm{cm}^{2}$. Vinculin staining experiments to visualize the focal adhesions revealed a difference in cell-adhesion between the PHEMA and the PPEGMA based substrates. This difference was tentatively attributed to the enhanced water-solubility of the PPEGMA-brushes as compared to the PHEMA-brushes in combination with the longer oligoethylene glycol spacer that separates the polymer backbone and the RGD peptide in the case of the PPEGMA brushes. In addition, cells on the brushes were stable to exposure of a shear stress of 15 dynes $/ \mathrm{cm}^{2}$, which served to simulate arterial blood flow, for a period of 24 h. Taken together, these results provided a proof-of-concept for the feasibility of polymer brushes produced via SI-ATRP as a platform for the development of bioactive surface coatings.

Another area of application that provides many attractive opportunities for the use of SI-ATRP is biosensing, e.g. in protein microarrays. Protein microarrays are powerful tools allowing the measurement of protein-protein interactions, protein abundances or protein modifications. ${ }^{[3,4]}$ Protein microarrays require chips with binding surfaces that allow the immobilization of proteins at high but controlled surface concentrations to enable multiplex analysis. Protein chip substrates can either 


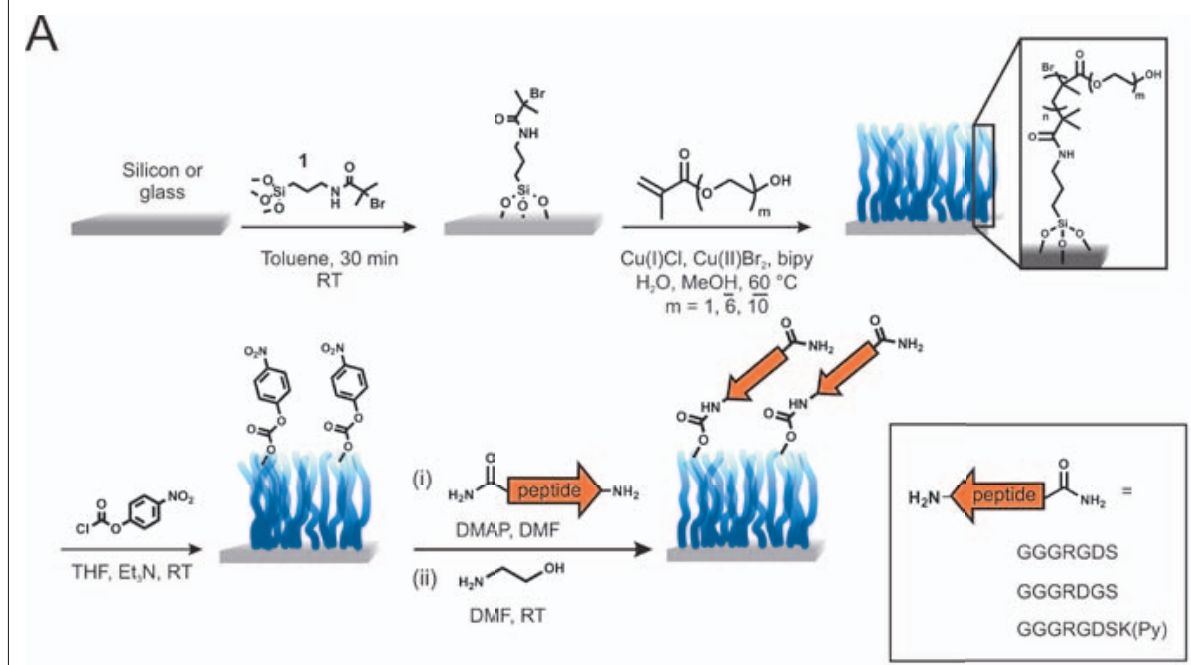

B

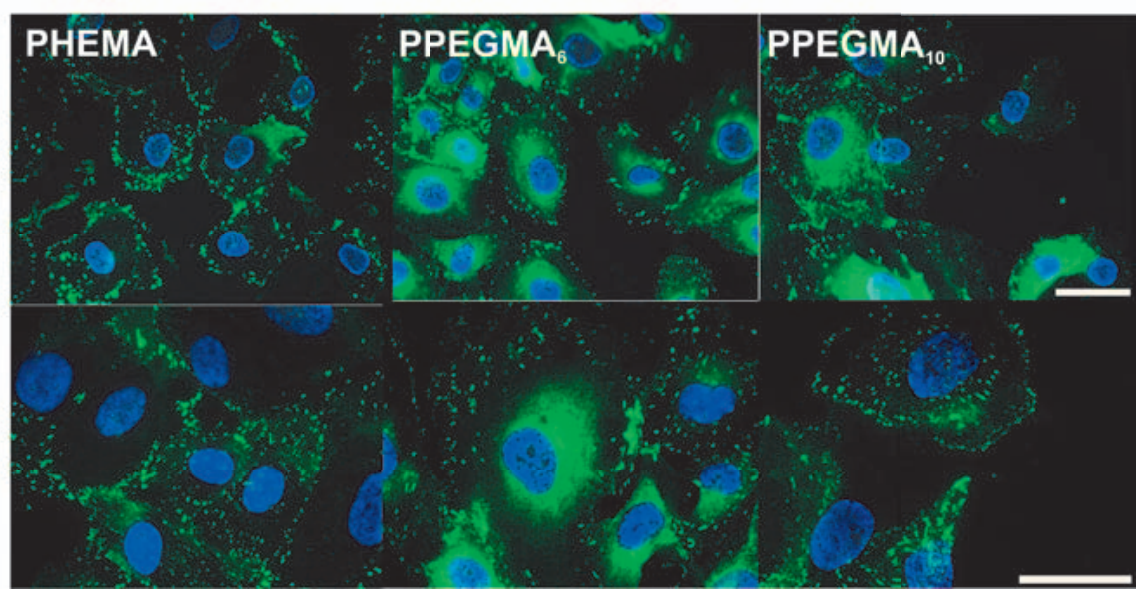

Fig. 1. (A) Synthesis of RGD modified PHEMA and PPEGMA brushes via SI-ATRP; (B) Fluorescence micrographs of HUVECs 4h postseeding on $20 \mathrm{~nm}$ thick RGD functionalized polymer brushes after DAPI and vinculin staining to visualize cell nuclei and focal adhesions, respectively (scale bar 25 um) ${ }^{[2]}$ ). Reprinted from Biomaterials, 28, 2536-2546, Copyright 2007, with permission from Elsevier.

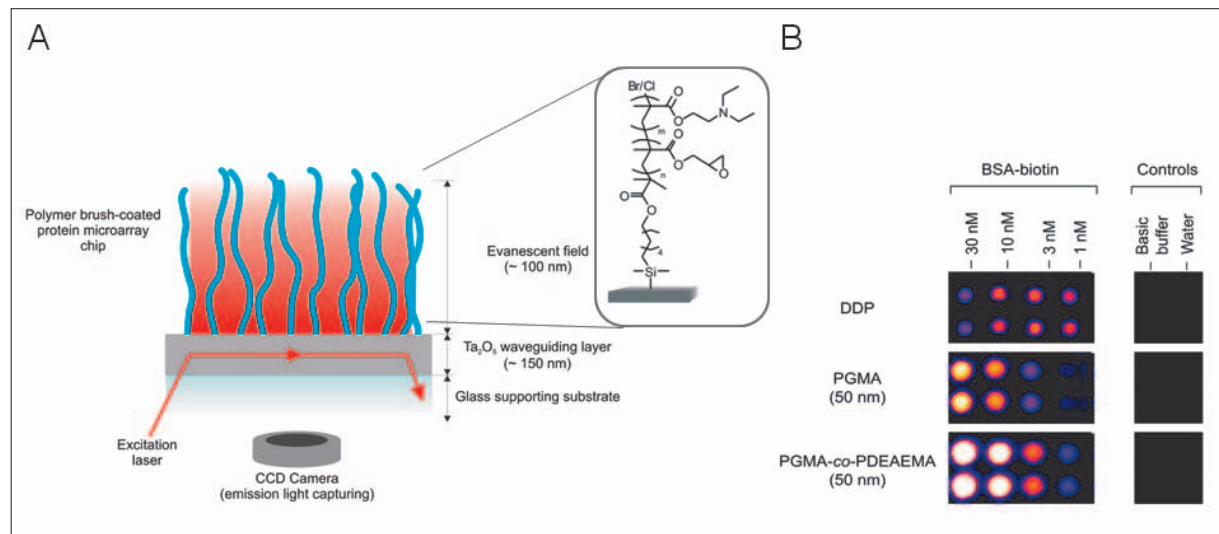

Fig. 2. (A) Schematic representation of a polymer-brush modified waveguide-based protein microchip; (B) Fluorescence images of a PGMA and PGMA-co-PDEAEMA modified waveguide chip as well as a DDP-modified chip after immobilization of BSA-biotin and subsequent exposure to AF-647 labelled streptavidin. Adapted with permission from ref. [9]. Copyright 2010 American Chemical Society.

be two-dimensional, e.g. dodecylphosphate (DDP), ${ }^{[4]}$ amine, ${ }^{[5]}$ or epoxide functionalized surfaces ${ }^{[5]}$ or three-dimensional, such as porous poly(vinylidenefluoride $)^{[6]}$ or nitrocellulose films, ${ }^{[7]}$ thin hydrogel coatings ${ }^{[8]}$ or a polymer brush. ${ }^{[9]}$ The main advantage of three-dimensional substrates is that they provide an increased protein binding capacity. An interesting example is poly(glycidyl methacrylate) (PGMA) and poly(glycidyl methacrylate)-copoly(2-(diethylamino)ethyl methacrylate (PGMA-co-PDEAEMA) brushes, which can be prepared via SI-ATRP. ${ }^{[9,10]}$ These brushes contain epoxide groups that can react with nucleophilic groups on proteins, e.g. lysine residues. The incorporation of a small fraction of DEAEMA in these brushes was not only found to increase the rate of the protein immobilization reaction, but also increased the overall protein binding capacity. ${ }^{[10]}$ In two model experiments, which included the detection of biotin-streptavidin binding as well as a model TNF $\alpha$ reverse assay, the 3D polymer brush substrates were compared to a DDP-modified 2D substrate (Fig. 2). These experiments were accomplished under real protein microarray conditions with an automated nanospotter and read-out system and demonstrated that the brushes were superior to the 2D DDP control not only in terms of absolute fluorescence read-outs, but also with regards to the signal-to-noise ratio. ${ }^{[9]}$

\section{Polymer Therapeutics}

The term polymer therapeutics covers a variety of nanosized pharmaceuticals ('nanomedicines') that includes macromolecular drugs, polymer-drug and polymerprotein conjugates as well as micelles containing covalently bound drugs. ${ }^{[11,12]}$ In these constructs, the polymer component can contribute in various ways to the overall therapeutic efficacy, for example by enhancing the stability of the drug towards premature degradation (which is particularly relevant in the case of sensitive biomolecular drugs), increasing plasma half-life or to allow passive targeting to tumor tissue (this is referred to as the enhanced permeability and retention (EPR) effect). ${ }^{[13]}$

A critical part of any polymer-drug conjugate is the linker that attaches the drug to the polymer backbone. This linker should be stable during circulation, but allow intracellular release of the drug. For this purpose a variety of linkers has been reported. ${ }^{[12]}$ Ester linkages or enzymatically cleavable peptide linkers have been most frequently used to attach anticancer drugs (such as doxorubicin or paclitaxel). ${ }^{[14]}$ In addition, $\mathrm{pH}$-sensitive covalent cis-acetonityl, hydrazone or acetal linkers, which are cleaved in the slightly acidic environment of the endosomal and lysosomal compartments have been reported. ${ }^{[12,14]}$ Other than releasing the payload at a specific subcellular compartment, however, the linkers that have been used so far do not play a further, active role in guiding the delivery process. An interesting alternative to such covalent polymer-drug conjugates would be noncovalent polymer therapeutics in which 
the drug molecules are bound to a polymer carrier via a coiled coil-based linker (Fig. $3) .{ }^{[15]}$ Capitalizing upon the membrane lytic properties of several coiled coils, ${ }^{[16,17]}$ non-covalent linkers can be envisioned that not only release the payload at a specific subcellular location, e.g. due to a change in $\mathrm{pH}$ upon trafficking from the extracellular milieu to the endosomes, but which also, after dissociation of the linker generate (a) peptide sequence(s) that is/are membrane active and facilitate(s) the escape of the payload from the endosome to the cytosol. The latter cannot be achieved using a conventional, covalent linker and would be a unique feature of the proposed non-covalent polymer-drug conjugates. Efficient translocation from the endosomal compartments to the cytosol is a critical step in the delivery of therapeutic bio(macro) molecules such as proteins, peptides, DNA and RNA. Efficient endosomal escape is important not only for these drugs to be able to exert their function in the cytosol, but also to prevent subsequent lysosomal degradation. ${ }^{[18]}$ As a first proof-of-concept, model non-covalent polymer-drug conjugates have been prepared that are based on the so-called E3/K3 motif. ${ }^{[19]}$ It is important to point out that the E3/K3 linker does not possess any biological activity, but mainly serves as a model system to investigate whether an appropriately designed coiled coil linker could be used to non-covalently carry a payload and release this upon a change in $\mathrm{pH}$ as the constructs enter the endosomal intracellular compartments. The E3/K3 motif was chosen since this pair of peptides was found to form a stable heterodimeric superhelix at pH 7 and undergoes an unfolding transition at acidic $\mathrm{pH}$ values reminiscent of the endosomal compartments. ${ }^{[20]}$ Preliminary cell experiments provided a first proof-ofconcept and indeed suggested that the E3/ $\mathrm{K} 3$ motif is a suitable non-covalent linker that allows to attach cargo in biologically relevant media, that the polymer-drug conjugates are taken up by the cells and that release of the guest indeed seems to occur within the cell in response to a drop in $\mathrm{pH}$ that the constructs experience when they traffic from the extracellular milieu to the intracellular compartments. ${ }^{[15]}$

In addition to their use as scaffolds to attach and subsequently release drugs, polymers are also used in nanomedicine to overcome problems related to the stability or immunogenicity of the drug of interest. This is particularly relevant for biomolecular drugs such as peptides and proteins, which are susceptible to enzymatic degradation. The most successful approach that has been used to date to overcome these limitations involves the modification of the peptide or protein of interest with poly(ethylene glycol) (PEGylation). ${ }^{[21,22]}$

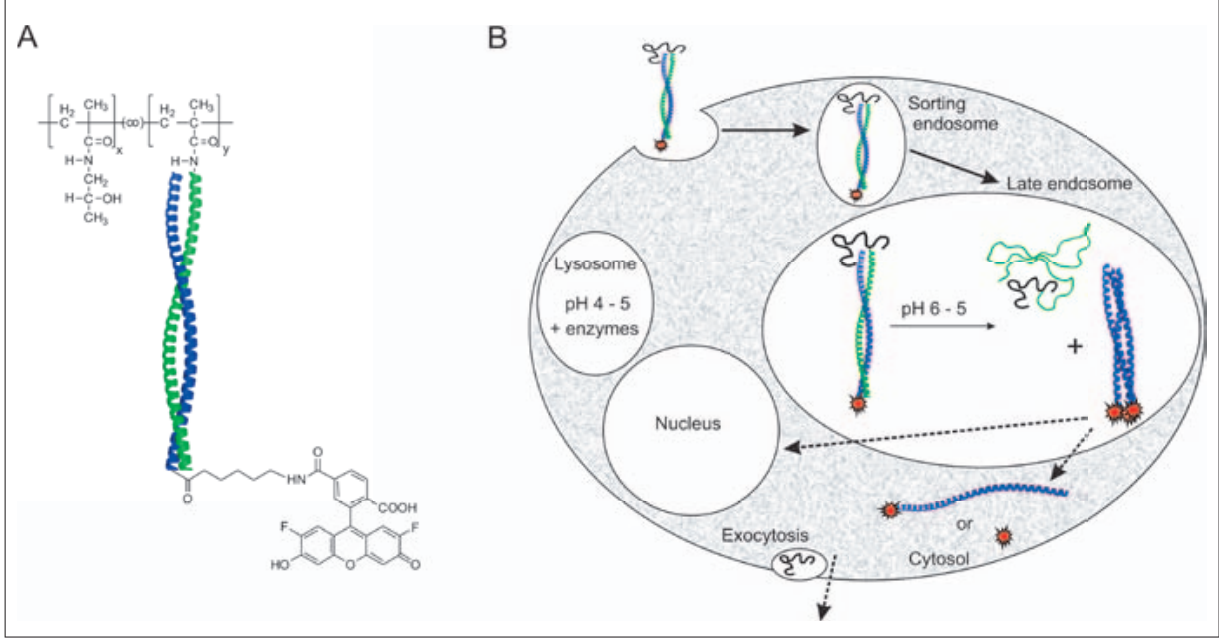

Fig. 3. (A) Structure of the proposed non-covalent polymer therapeutics. In this illustration, a fluorescent dye, Oregon Green, instead of a real drug molecule is used to represent the cargo that can be transported and released. (B) Envisioned pathway for the cell uptake of the proposed noncovalent polymer therapeutics and subsequent intracellular release of the cargo. Reprinted with permission from ref. [15]. Copyright 2010 American Chemical Society.

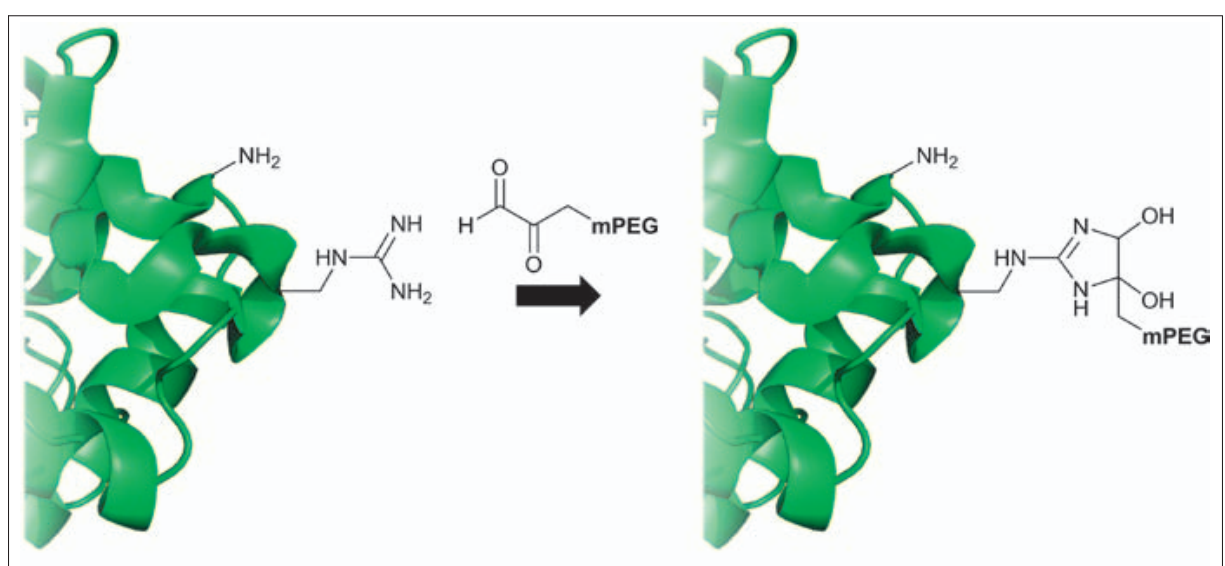

Fig. 4. Selective conjugation of $\alpha$-oxo-aldehyde PEG to arginine residues. Reprinted with permission from ref. [26]. Copyright 2011 American Chemical Society.

First-generation PEGylation strategies generally involved the use of linear PEGderivatives with a functional end group that can undergo reaction with any of the side chain or terminal amine groups of the protein. ${ }^{[21,22]}$ While often being successful in terms of enhancing the stability and/or reducing the immunogenicity of the protein of interest, first-generation PEGylation is frequently plagued by loss of activity of the protein. One probable reason for this is the lack of selectivity during the modification reaction. To overcome these limitations, there is an interest in exploring alternative polymer architectures ('branched PEG') and the development of novel site-selective PEGylation reactions. ${ }^{[23-25]}$ Recently, the toolbox for site-selective protein modifications was enlarged by the development of arginine-selective $\alpha$-oxoaldehyde modified PEGs (Fig. 4). ${ }^{[26]}$ This conjugation strategy is inspired by the Maillard reaction, which involves the glycation of amino groups of a protein with reducing sugars or endogeneous aldehydes or ketones. While the reaction can take place with lysine or arginine residues, only the arginine adducts are stable, i.e. a 'thermodynamic selectivity' towards arginine residues is achieved. This was proven by model reactions and possible cleavage of the lysine-conjugates with hydroxylamine. This approach was recently extended to other polymers than PEG, which are accessible via atom transfer radical polymerization by selective initiation with a protected $\alpha$-oxo-aldehyde.[27]

\section{Conclusions}

The examples discussed in this contribution serve to illustrate the potential of modern polymer synthesis strategies as enabling tools to address problems at the biology interface. While the present contribution represents a subjective selection of examples from the author's own work, 
it is obvious that the scope of the methods discussed goes beyond the application areas covered herein. With the ever increasing possibilities to synthesize polymers with precisely defined molecular weight, architecture and functionality and the continuous emergence of novel strategies to residue- and site-selectively conjugate biological and synthetic polymers, the scope and utility of this toolbox will undoubtedly further expand.

Received: May 26, 2011

[1] R. Barbey, L. Lavanant, D. Paripovic, N Schüwer, C. Sugnaux, S. Tugulu, H.-A. Klok, Chem. Rev. 2009, 109, 5437.

[2] S. Tugulu, P. Silacci, N. Stergiopulos, H.-A Klok, Biomaterials 2007, 28, 2536.

[3] P. Mitchell, Nat. Biotech. 2002, 20, 225.

[4] M. Pawlak, E. Schick, M. A. Bopp, M. J. Schneider, P. Oroszlan, M. Ehrat, Proteomics 2002, 2, 383.

[5] K. Martin, T. H. Steinberg, L. A. Cooley, K. R. Gee, J. M. Beechem, W. F. Patton, Proteomics 2003, 3, 1244

[6] A. Lueking, M. Horn, H. Eickhoff, K. Büssow, H. Lehrach, G. Walter, Anal. Biochem. 1999, 270, 103.

[7] B. A. Stillman, J. L. Tonkinson, BioTechniques 2000, 29, 630

[8] P. Arenkov, A. Kukhtin, A. Gemmell, S. Voloshchuk, V. Chupeeva, A. Mirzabekov, Anal. Biochem. 2000, 278, 123.

[9] R. Barbey, E. Kauffmann, M. Ehrat, H.-A. Klok, Biomacromolecules 2010, 11, 3467.

[10] R. Barbey, H.-A. Klok, Langmuir 2010, 26, 18219.
[11] R. Haag, F. Kratz, Angew. Chem. Int. Ed. 2006, 45, 1198.

[12] R. Duncan, Nat. Rev. Drug. Discov. 2003, 2, 347.

[13] H. Maeda, J. Wu, T. Sawa, Y. Matsumura, K. Hori, J. Control. Release 2000, 65, 271.

[14] R. Duncan, Nat. Rev. Cancer 2006, 6, 688.

[15] B. Apostolovic, S. P. E. Deacon, R. Duncan, H. A. Klok, Biomacromolecules 2010, 11, 1187.

[16] K. J. Cross, W. A. Langley, R. J. Russell, J. J. Skehel, D. A. Steinhauer, Protein Pept. Lett. 2009, 16, 766 .

[17] A. K. Gillingham, S. Munro, Biochim. Biophys. Acta-Mol. Cell Res. 2003, 1641, 71.

[18] I. Nakase, S. Kobayashi, S. Futaki, Biopolymers 2010, 94,763

[19] J. R. Litowski, R. S. Hodges, J. Biol. Chem. 2002, 277, 37272 .

[20] B.Apostolovic, H.-A. Klok, Biomacromolecules 2008, 9, 3173.

[21] F. M. Veronese, Biomaterials 2001, 22, 405.

[22] M. J. Roberts, M. D. Bentley, J. M. Harris, $A d v$. Drug Deliv. Rev. 2002, 54, 459.

[23] F. Wurm, J. Klos, H. J. Räder, H. Frey, J. Am. Chem. Soc. 2009, 131, 7954.

[24] M. A. Gauthier, H.-A. Klok, Chem. Commun. 2008, 2591.

[25] F. M. Veronese, P. Caliceti, O. Schiavon, J. Bioact. Compatible Polym. 1997, 12, 196.

[26] M.A. Gauthier, H.-A. Klok, Biomacromolecules 2011, 12, 482 .

[27] M. A. Gauthier, M. Ayer, J. Kowal, F. R. Wurm, H.-A. Klok, Polym. Chem. 2011, 2, 1490. 